\title{
THE
}

$5-1-1984$

\section{Comment on "Ground-State Properties of a Spin-1 Antiferromagnetic Chain"}

Jill C. Bonner

University of Rhode Island

Gerhard Müller

University of Rhode Island, gmuller@uri.edu

Follow this and additional works at: https://digitalcommons.uri.edu/phys_facpubs

Terms of Use

All rights reserved under copyright.

\section{Citation/Publisher Attribution}

Bonner, J. C. \& Müller, G. Comment on "ground state properties of a spin-1 antiferromagnetic chain. Phys. Rev. B 29 (1984), 5216-5221.

Available at: http://dx.doi.org/10.1103/PhysRevB.29.5216

This Article is brought to you for free and open access by the Physics at DigitalCommons@URI. It has been accepted for inclusion in Physics Faculty Publications by an authorized administrator of DigitalCommons@URI. For more information, please contact digitalcommons-group@uri.edu. 


\title{
Comments
}

Comments are short papers which comment on papers of other authors previously published in the Physical Review. Each Comment should state clearly to which paper it refers and must be accompanied by a brief abstract. The same publication schedule as for regular articles is followed, and page proofs are sent to authors.

\section{Comment on "Ground-state properties of a spin-1 antiferromagnetic chain"}

\author{
Jill C. Bonner \\ Department of Physics, University of Rhode Island, Kingston, Rhode Island 02881 \\ Gerhard Müller \\ Institute for Theoretical Physics, State University of New York at Stony Brook, \\ Stony Brook, New York 11794
}

(Received 11 April 1983)

\begin{abstract}
Botet and Jullien [Phys. Rev. B 27, 613 (1983)] have conducted a finite-chain scaling analysis on the spin-1 antiferromagnetic Heisenberg-Ising chain. They claim that the ground-state properties are completely different from the case of spin $\frac{1}{2}$. Using finite-size scaling techniques, they conclude that between the $X Y$ gapless phase and the doublet-ground-state Néel phase, a new phase appears in an extended anisotropy range. This phase, which encompasses the isotropic Heisenberg model, is characterized by a nonmagnetic singlet ground state and a nonzero excitation-energy gap. We perform a similar finite-chain scaling analysis on the spin- $\frac{1}{2}$ antiferromagnetic Heisenberg-Ising chain and obtain strikingly similar results. Since the spin- $\frac{1}{2}$ system is known rigorously not to exhibit the type of behavior proposed by Botet and Jullien, their analysis is open to doubt. The possibility arises that a strong singularity at the Heisenberg point for spin 1 (similar to the essential singularity for spin $\frac{1}{2}$ ) may be giving rise to misleading results. The $T=0$ phase behavior of the spin-1 Heisenberg-Ising antiferromagnetic chain, therefore, remains in question and specific additional numerical studies are proposed to clarify the matter.
\end{abstract}

\section{INTRODUCTION}

Following recent theoretical work of Haldane, ${ }^{1-3}$ which suggests that very different $T=0$ phase behavior will be observed in the one-dimensional (1D) Heisenberg-Ising antiferromagnet (AFM)

$$
H=2 \sum_{l=1}^{N}\left(S_{l}^{x} S_{l+1}^{x}+S_{l}^{y} S_{l+1}^{y}+\lambda S_{l}^{z} S_{l+1}^{z}\right), \quad \lambda \geqslant 0,
$$

depending on whether the spin is integral or half-integral, Botet and Jullien (BJ) have investigated in detail the $T=0$ properties of Hamiltonian (1) with $S=1$ by means of finite-chain calculations. ${ }^{4}$ They interpret their results as supporting Haldane's conclusions, which are summarized in what follows.

Special cases of Hamiltonian (1) are the $X Y$ model $(\lambda=0)$, the Heisenberg model $(\lambda=1)$, and the Ising model $(\lambda=\infty)$. The situation $0 \leqslant \lambda<1$ corresponds to easy-plane anisotropy, and $\lambda>1$ to easy-axis anisotropy. For $S=\frac{1}{2}$, the $T=0$ phase behavior of (1) is well known: ${ }^{5-7}$ For $0 \leqslant \lambda \leqslant 1$ the $X X Z$ model (1) has a singlet $\left(S_{z}^{\text {tot }}=0\right)$ ground state (GS) and a gapless excitation spectrum. There is no true long-range order (LRO) in the GS. The two-spin correlation functions decay asymptotically for large distances as a power law: ${ }^{8}$

$$
\begin{aligned}
& \left\langle S_{l}^{x} S_{l+R}^{x}\right\rangle \sim(-1)^{R} / R^{-1+\eta_{x}}, \quad-1+\eta_{x}=1-\mu / \pi, \\
& \left\langle S_{l}^{z} S_{l+R}^{z}\right\rangle \sim(-1)^{R} / R^{-1+\eta_{z}}, \quad-1+\eta_{z}=(1-\mu / \pi)^{-1},
\end{aligned}
$$

where $\lambda=\cos \mu, 0 \leqslant \mu \leqslant \pi / 2$. The Heisenberg model $(\lambda=1)$ marks the termination of the gapless regime [see Fig. 1(a)]. For $\lambda>1$, the system has a twofold degenerate GS separated from the excitation spectrum by a gap whose magnitude is given by ${ }^{5-7}$

$$
\Delta=\frac{\pi \sinh x}{x} \sum_{n=-\infty}^{+\infty} \operatorname{sech}\left[(2 n+1) \pi^{2} / 2 x\right],
$$

where $\cosh x=\lambda \geqslant 1$. For $\lambda \rightarrow 1$, the expression behaves as

$$
\Delta \sim 4 \pi \exp \left[-\pi^{2} / \sqrt{2(\lambda-1)}\right] \text {. }
$$

In this regime, the system has a nonzero sublattice magnetization given by ${ }^{9}$

$$
M_{z}^{2}=\lim _{R \rightarrow \infty}\left|\left\langle S_{l}^{z} S_{l+R}^{z}\right\rangle\right|=\frac{2 \pi}{x}\left[\sum_{n=1}^{\infty} \exp \left(-\left(n-\frac{1}{2}\right)^{2} \frac{\pi^{2}}{2 x}\right)\right]^{2} .
$$

Note that both $\Delta$ and $M_{z}$ vanish exponentially as $\lambda \rightarrow 1$. In fact, the point $\lambda=1$ is the location of an essential singularity for these and also for other $T=0$ quantities. In the Ising limit $(\lambda \rightarrow \infty)$ the GS doublet can be identified as the pair of Néel states $|\uparrow \downarrow \uparrow \cdots \downarrow\rangle,|\downarrow \uparrow \downarrow \cdots \uparrow\rangle .^{10}$ Here $M_{z}$ reaches its saturation value. According to Haldane, ${ }^{1}$ such behavior is characteristic of Hamiltonian (1) with arbitrary half-integral spin.

For $S=1$, on the other hand, BJ claim to have found a $T=0$ phase behavior which differs drastically from the one described above. According to Haldane, this new behavior 

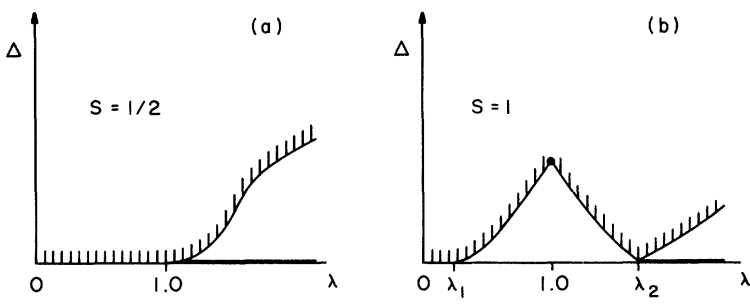

FIG. 1. Energy gap $\Delta$ between the ground state and the excitation spectrum of the Heisenberg-Ising antiferromagnet [Eq. (1)] as a function of anisotropy $\lambda$ ( schematic). Part (a) is based on rigorous results for $S=\frac{1}{2}$ : The region $0 \leqslant \lambda \leqslant 1$ is gapless. The Heisenberg point, $\lambda=1$, is the location of an essential singularity marking a transition to a phase with a doublet ground state and a nonzero excitation gap for $\lambda>1$. Part (b) illustrates the behavior of the case $S=1$ as conjectured by Haldane (Ref. 1) and supported by the finite-chain scaling analysis of Botet and Jullien (Ref. 4): Here the gapless region $\left(0 \leqslant \lambda<\lambda_{1}\right)$ and the region with doublet ground state and gap $\left(\lambda>\lambda_{2}\right)$ are separated by an intermediate phase $\left(\lambda_{1}<\lambda<\lambda_{2}\right)$ encompassing the Heisenberg point $\lambda=1$, characterized by a singlet ground state and a gap. According to this picture the symmetry change of the Hamiltonian [Eq. (1)] at $\lambda=1$ is manifested only in the crossing of the first (doublet) excited state for $\lambda<1$ and the first (singlet) excited state for $\lambda>1$ to form an $S^{\text {tot }}=1$ triplet (solid circle). In Ref. 4 it is conjectured that $\lambda_{1}=0$ i.e., the gapless phase exists only for $\lambda=0$.

should be characteristic of Hamiltonian (1) for arbitrary integral spin. In this case, both the gapless $X Y$-like phase without true LRO and the ordered Ising-like phase with a doublet GS and an excitation gap still occur. However, instead of a transition between the two phases occurring at the Heisenberg point, $\lambda=1$, where $H$ changes symmetry, an intermediate phase is conjectured in an extended parameter region between $\lambda_{1}<1$ and $\lambda_{2}>1$, which encompasses the Heisenberg point [see Fig. 1(b)]. The intermediate phase is characterized by a nondegenerate GS separated by a gap from the excited states. There is no LRO. At $T=0$ the correlation functions decay exponentially to zero for large distances. According to Refs. 1 and 4, the in-plane correlation function $\left\langle S_{l}^{x} S_{l+R}^{x}\right\rangle$ at $\lambda=\lambda_{1}$ and the out-of-plane correlation function $\left\langle S_{l}^{z} S_{l+R}^{z}\right\rangle$ at $\lambda=\lambda_{2}$ both decay asymptotically with the same power law, i.e., as $(-1)^{R} / R^{1 / 4}$.

This conjectured phase behavior of the $S=1$ HeisenbergIsing AFM appears to be in contradiction to general principles in the theory of phase transitions and critical phenomena based on symmetry and continuity considerations which go by the names of smoothness ${ }^{11}$ and universality. ${ }^{12}$ Specifically, the conclusions of BJ for the $S=1$ Heisenberg-Ising AFM are that, at some intermediate point $\lambda_{2}$ in the easyaxis regime, the critical properties of the system change drastically, whereas the symmetry character of Hamiltonian (1) does not. A similar situation occurs at $\lambda_{1}$ in the easyplane regime. At $\lambda=1$, on the other hand, where $H$ changes from easy-plane symmetry to easy-axis symmetry, the $T=0$ properties show no singular character whatsoever.

We might note here that critical singularities or phase transitions occuring at $\lambda \neq 1$ have been predicted rather commonly in the past for the 1D $S=\frac{1}{2}$ Heisenberg-Ising AFM on the basis of various approximate calculations. ${ }^{13}$ Some degree of confusion resulted until numerical calculations ${ }^{14}$ followed by rigorous analytic calculations ${ }^{5,7,15}$ finally made it clear that $\lambda=1$ is the only singular point. ${ }^{16}$
The BJ picture is also in conflict with a large body of work involving numerical studies on Heisenberg chains with $S>\frac{1}{2} .{ }^{17,18}$ Previous workers have concluded that Heisenberg spin chains have qualitatively the same critical behavior for arbitrary spin. Further, they have made the observation that their results are in increasingly better agreement with Kubo spin-wave theory, ${ }^{18,19}$ as the spin value increases. Since the numerical work of BJ on spin-1 chains is the first to contradict the accepted understanding, it seems appropriate to apply a close scrutiny.

\section{FINITE-SIZE SCALING ANALYSIS OF NUMERICAL RESULTS}

A major goal of previous numerical studies on spin-1 Heisenberg chains has been to generate curves for the thermodynamic properties and then extrapolate to the thermodynamic limit for subsequent comparison with experiment. Hence it has been necessary to calculate all the $(2 S+1)^{N}$ eigenvalues for the finite systems, restricting the maximum system size to 7 or 8 . Since the goal of BJ is the verification of Haldane's prediction, they are interested only in the two lowest-lying energy states. Consequently, they have been able to exploit the powerful Lanczös algorithm to generate such states for systems of $N=2$ through 12 spins (they consider only even $N$ ). Hence the BJ results represent a significant advance on previous work in terms of information on the low-lying states.

Botet and Jullien find that for finite chains (with periodic boundary conditions) the GS is always a nondegenerate $S_{z}^{\text {tot }}=0$ singlet. For $0 \leqslant \lambda<1$, the first excited state is an $S_{z}^{\text {tot }}= \pm 1$ doublet, and for $1<\lambda<\infty$, the first excited state is an $S_{z}^{\text {tot }}=0$ singlet. At the Heisenberg point, $\lambda=1$, the singlet and doublet states cross to yield a threefold degenerate, total spin $S^{\text {tot }}=1$, triplet. The question which BJ address is how the energy gap $G_{N}$ between the two lowest eigenstates behaves as a function of $N$, i.e., whether it persists or disappears in the thermodynamic limit, $N \rightarrow \infty .{ }^{20}$ In a finite-size scaling context, the limiting behavior is studied by plotting the so-called "scaled gaps" $N G_{N}$ as a function of $\lambda$. The results are shown in Fig. 1 of Ref. 4. At $\lambda=0$, the $N G_{N}$ appear to be independent of $N$, which BJ correctly interpret as indicating that $G_{N}$ vanishes as $1 / N$ $(N \rightarrow \infty)$ for the spin-1 $X Y$ model. For $0<\lambda \leqslant 1$, the values of $N G_{N}$ at fixed $\lambda$ show a smooth upward trend with $N$, and this trend reaches a maximum at $\lambda=1$. BJ apparently take the view that the nonsuperposition and upward trend of $N G_{N}$ indicate a divergence of $N G_{N}$ as $N \rightarrow \infty$, i.e., that the region $0<\lambda \leqslant 1$ has a nonzero excitation gap $G_{\infty}$ $=\Delta>0$ in the thermodynamic limit. This is interpreted by BJ as supporting Haldane's picture (with $\lambda_{1}=0$ ) of an intermediate phase with singlet-GS and nonzero excitation gap $\Delta$. The correct way to approach this situation is, however, not just to look whether the $N G_{N}$ superpose, but whether they converge to some finite limiting value as $N \rightarrow \infty$. If $\lim _{N \rightarrow \infty} N G_{N}$ is finite and nonzero, $G_{N}$ eventually vanishes as $1 / N$ characteristic of an excitation continuum closing on the GS (quasidegeneracy). If $\lim _{N \rightarrow \infty} N G_{N}=0$, then $G_{N}$ behaves asymptotically as $1 / N^{1+\epsilon}, \epsilon>0$, and this is characteristic of a truly degenerate GS and the presence of LRO. In order to have a nonzero $G_{\infty}$, i.e., a GS which is nondegenerate and separated by a gap from the excitation spectrum, $\lim _{N \rightarrow \infty} N G_{N}$ must be divergent. ${ }^{21}$ Care is required, 
however, since finite-size effects setting in for $\lambda>0$ will slow the convergence for small $N$. Botet and Jullien do not seem to have appreciated this important point, and their conclusions must be regarded as premature.

For $\lambda>1$, interesting behavior is observed with the relative ordering of the $N G_{N}$ curves as a function of $N$ inverting at a value of $\lambda=1.18$. Again, BJ interpret this phenomenon in the context of finite-size scaling theory by arguing, essentially, that the upward trend of the $N G_{N}$ curves for $1 \leqslant \lambda \leqslant 1.18$ indicates a nonzero $G_{\infty}$, which vanishes as $\lambda \rightarrow 1.18 .^{22}$ Botet and Jullien identify $\lambda \simeq 1.18$ as the termination point $\lambda_{2}$ of the intermediate phase predicted by Haldane. Correspondingly, the decreasing trend of the $N G_{N}$ curves for $\lambda \gtrsim 1.18$ is interpreted as reflecting the presence of a doublet GS in the limit $N \rightarrow \infty$. Here again, the interpretation by $\mathrm{BJ}$ of their finite-chain results is inadequate for two reasons:

(i) The analysis is based on the assumption that for $N \rightarrow \infty, N G_{N}$ either diverges or vanishes for all $\lambda$ except the extrapolated "crossing point" $\lambda_{2}$; the possibility of a convergence of $N G_{N}$ to a finite nonzero value in an extended $\lambda$ range is not taken into consideration.

(ii) The symmetries of Hamiltonian (1) strongly suggest that the $T=0$ phase transition occurs at $\lambda=1$. Moreover, the exact results for the $S=\frac{1}{2}$ case indicate that $\lambda=1$ might be the location of an essential singularity. Under such circumstances great care must be exercised in the interpretation of approximate calculations, for the presence of an essential singularity is well known to cause numerical extrapolations to yield misleading results.

Taking these possible complications into consideration it appears that the behavior of the $N G_{N}$ curves interpreted by BJ as being in strong support of Haldanes's predictions is consistent with the picture known to be exact for the $S=\frac{1}{2}$ chain: The $T=0$ critical behavior changes only at $\lambda=1$, where the symmetry of $H$ also changes.

In support of this alternative interpretation of the behavior of the $S=1$ finite-chain results, an investigation of the corresponding behavior of $S=\frac{1}{2}$ finite chains is presented and discussed. For $S=\frac{1}{2}$ and $\lambda \geqslant 1$, calculations were carried out by one of us for chains with 2 through 10 spins and were discussed in detail some time ago. ${ }^{14}$ More recently they have been augmented by results for $N=12$ without affecting the original discussion. ${ }^{23}$ The results for $\lambda \geqslant 1$, plotted in the same way as those of BJ, are displayed in Fig. 2. Note that there is a striking qualitative resemblance between Fig. 1 of BJ and our Fig. 2. In particular, the curves $N G_{N}$ cross at values of $\lambda_{c}=1.1-1.16$. This is in strong resemblance to the situation for $S=1$ with one exception. For $S=1$, the scaled gaps $N G_{N}$ at $\lambda=1$ show a more divergent tendency than their $S=\frac{1}{2}$ counterparts, and hence $\lambda_{c}$ has an increasing trend with $N$ for small systems. If this tendency persists for large $N$, and goes as fast as $N$, then the $S=1$ Heisenberg AFM chain has a gap. However, as will be discussed later, if $\lambda=1$ corresponds to a singularity, as in the case of $S=\frac{1}{2}$, then $\lambda_{c}$ extrapolations can be misleading.

By the arguments of BJ, Fig. 2 could be interpreted as reflecting the existence of a critical anisotropy $\lambda_{2} \simeq 1.075$ where the $T=0$ critical behavior changes from a phase with singlet GS and nonzero excitation gap to phase with doublet GS. Since this is known rigorously not to be the case of $S=\frac{1}{2}$, the conclusion presents itself that the behavior of the spin-1 chain may be very similar to the behavior of the

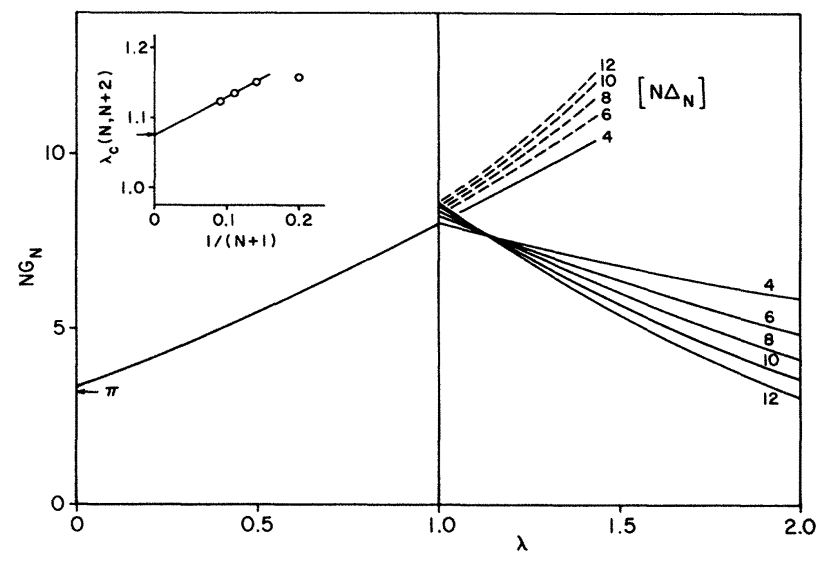

FIG. 2. Plot of the scaled gaps as a function of anisotropy for spin $\frac{1}{2}$. Data for $N=4,6,8,10,12$ are available for $\lambda \geqslant 1$ for both $N G_{N}$ and the "true" scaled gaps $N \Delta_{N}$ for $0<\lambda<1$, we have data only for $N=4$. The crossing points of $N G_{N}$ for $\lambda>1$, denoted $\lambda_{c}(N, N+2)$, are plotted vs $1 /(N+1)$ in the inset. The $\lambda_{c}$ appear to extrapolate to a limiting value of 1.075 , i.e., $7.5 \%$ higher than the exact value $\lambda_{c}=1$.

spin- $\frac{1}{2}$ chain, and, by extension, that integer spin chains show excitation spectra and $T=0$ critical properties which are analogous to those of half-integer spin chains. This is in agreement with continuity and symmetry considerations, which might be expected to apply to Heisenberg spin chains with arbitrary spin.

It is interesting to carry the discussion further. For $0<\lambda<1$, we have available exact results only for $G_{4}$ for $S=\frac{1}{2}$ as shown in Fig. 2. In addition, we know the exact result $G_{N}=2 \tan (\pi / 2 N)$ for $\lambda=0$ and $N$ even. ${ }^{24}$ Hence, at $\lambda=0$ the $N G_{N}$ converge very rapidly to the limit $\pi$ with correction terms $O\left(N^{-2}\right)$. This is characteristic for a system which behaves regularly. Rigorous results indeed confirm that $\lambda=0$ is not a singular point of the $S=\frac{1}{2} X X Z$ model GS. At $\lambda \simeq 1$, on the other hand, the convergence of $N G_{N}$ is found to be very much slower. This can be seen in Fig. 2 from the dashed curves, which represent the scaled singletdoublet gaps as continued into the region $\lambda \geqslant 1$ where we have data. ${ }^{25}$ Such slow convergence of finite-chain data is a characteristic feature of finite-chain data in the vicinity of an essential singularity. For $S=\frac{1}{2}, \lambda=1$ is indeed the location of an essential singularity.

According to Fig. 1 of Ref. 4 , the $S=1$ finite-chain data exhibit similar behavior: The convergence of $N G_{N}$ is very rapid at $\lambda=0$ and extremely slow (or possibly nonexistent) at $\lambda=1$. The interpretation of $\mathrm{BJ}$ is, however, exactly the opposite of the interpretation which is correct for $S=\frac{1}{2}$ : The very rapid convergence of the $N G_{N}$ at $\lambda \simeq 0$ is attributed to the presence of an essential singularity at $\lambda=0$, whereas the extremely slow convergence or apparent divergence of $N G_{N}$ at $\lambda=1$ is interpreted as the regular behavior of a system with a nonzero excitation gap.

A point of some interest is that for $S=\frac{1}{2}$ and even $N$, the $N G_{N}$ converge monotonically from above at $\lambda=0$, whereas the convergence with $N$ is monotonic from below at $\lambda=1$. Hence, the $N G_{N}$ curves must successively cross at some intermediate $\lambda$. Such a crossing of scaled gap curves has not previously been observed in finite-size scaling treat- 
ments of models expected to show a gapless line of critical points ending in an essential singularity. ${ }^{26}$ The results for $S=1$, as displayed in Ref. 4, leave it unclear whether $N G_{N}$ at $\lambda=0$ converges from above or from below or whether the points superpose exactly.

Returning to discuss the phase transition at $\lambda_{2}$, we show in Fig. 3 the scaled gaps for $S=\frac{1}{2}$ chains as a function of $1 / N$ for various values of $\lambda$ in the vicinity of the Heisenberg point $\lambda=1$. Figure 3 is closely related to Fig. 24 of Ref. 14 . For $\lambda=1$, the values of $N G_{N}$ successively increase with $N$ in a manner which suggests a nonzero limiting value. However, an extrapolation of the points for $N=4,6, \ldots, 12$ would yield a result about $10 \%$ in error, i.e., $10 \%$ different than what is now known to be the exact result (indicated by an arrow in Fig. 3). Clearly, this situation is not amenable to a routine $1 / N$ extrapolation procedure, and no such attempt was made in Ref. 14. For $1<\lambda \leq 1.1$ the values of $N G_{N}$ again increase with $N$, although more slowly. A "reasonable" conclusion based on results for $N$ $=4,6, \ldots, 12$ would be that again $N G_{N}$ leads to a nonzero value in the limit $N \rightarrow \infty$. This is known rigorously not to be the case, and the implication is that for larger $N$ (say, $N \geq 30$ ) the curve for $N G_{N}$ has to turn downwards and go to zero in the limit $N \rightarrow \infty,{ }^{27}$ as indicated by the dashed lines (sketches) in Fig. 3. For curves corresponding to $\lambda \geq 2.0$ (not shown in Fig. 3), the downward trend is sufficiently well developed to indicate a zero limit, even for small $N$. It is clear that a corresponding plot for $S=1$

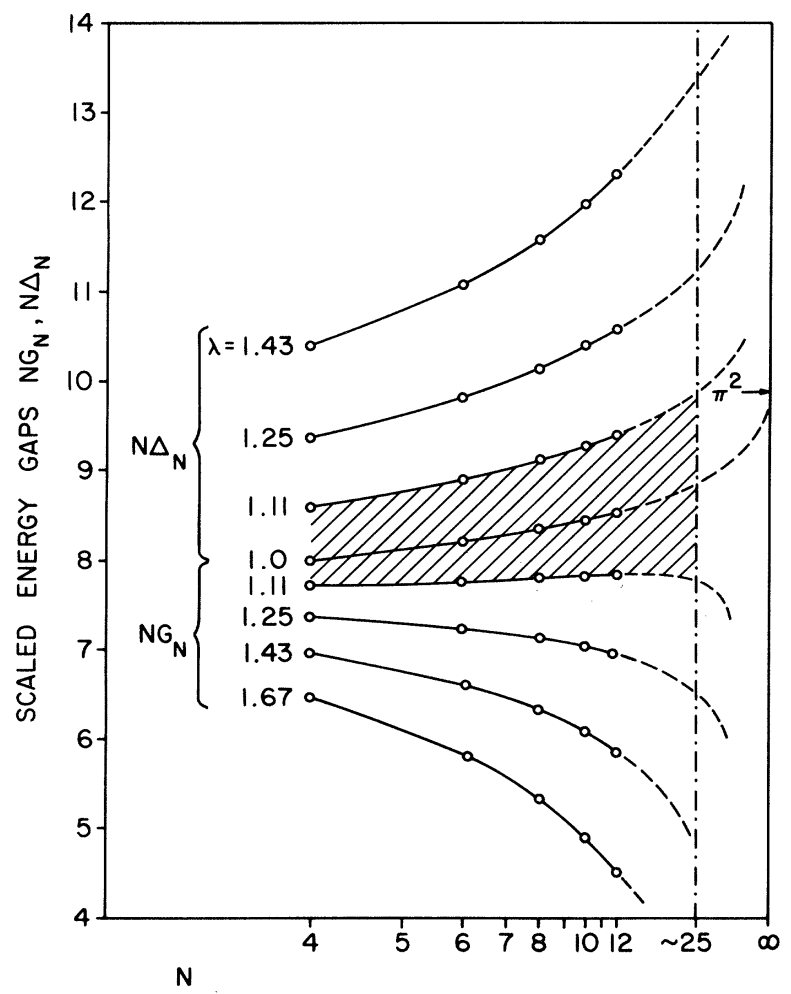

FIG. 3. Plot of the scaled gaps $N G_{N}$ and $N \Delta_{N}$ for $S=\frac{1}{2}$ and $N=4,6,8,10,12$ as a function of $1 / N$ for $\lambda$ values in the vicinity of $\lambda=1$. The shaded region, corresponding to a variation in $\lambda$ of $\Delta \lambda \simeq 0.1$, indicates a region where the large- $N$ limiting behavior is not apparent for systems smaller than $N=25$ (indicated by the dot-dashed line). The exact limiting value of $N G_{N}$ (and $N \Delta_{N}$ ) for $\lambda=1$ has the value $\pi^{2}$ as indicated by an arrow. would shown very similar features, and the apparent trend of points for $N G_{N}$ in the region $1 \leqslant \lambda \leqslant 1.18$ towards finite nonzero or even infinite limit would support the conclusion by $\mathrm{BJ}$ of a change in critical behavior at $\lambda \simeq 1.18$ rather than $\lambda=1$. This conclusion could be spurious, an artifact of very small systems in a region bordering a singularity. To obtain a more accurate picture of what happens in the limit $N \rightarrow \infty$, the $S=\frac{1}{2}$ results suggest that it might be necessary to study $S=1$ chains of at least 20-30 spins, a project beyond the capacity of present generation computers.

For comparison, and for illustrative purposes, the "true" finite system scaled excitation gaps for $\lambda>1$, denoted $N \Delta_{N}$, are shown also in Fig. 3. Such curves, for $\lambda>1$, must ultimately diverge as $N$, though this is not readily apparent for small systems, $N=4,6, \ldots, 12$, for $1<\lambda \leqslant 1.1 .^{28}$ The shaded region, corresponding to a spread in $\lambda$ values of $\Delta \lambda=0.1$, indicates a parameter range in which it is difficult to draw conclusions concerning the thermodynamic limit if one only has results available for small finite systems. For $S=1$ this $\lambda$ region is likely to include the "critical" value of BJ of $\lambda=1.18$. We conclude that close to a singular value of $\lambda$, a finite-size scaling analysis has to be performed with extreme caution.

\section{III. $T=0$ SINGULARITIES AND EXCITATION SPECTRUM}

The analysis above has served to establish the fact that the essential singularity of the $S=\frac{1}{2}$ Heisenberg-Ising AFM at $\lambda=1$ will result in misleading finite-chain extrapolations if the interpretation is too simplistic. It raises the strong possibility that a similar singularity occurs at $\lambda=1$ in spin-1 chains. As noted above, BJ do consider the possibility of an essential singularity, but conclude it must occur at $\lambda=0$. However, the minimal corrections to the $1 / N$ behavior of the gap energies at $\lambda=0$ for both $S=\frac{1}{2}$ and $S=1$, even for the smallest systems, is, in fact, an indication that the $X X Z$ model behaves regularly there. This has been verified by rigorous calculations for $S=\frac{1}{2}$, and is strongly suggested by the numerical calculations of BJ themselves for $S=1$.

According to $\mathrm{BJ}$, the $T=0$ phase transition which they believe to occur at $\lambda=\lambda_{2} \simeq 1.18$ is an ordinary second-order phase transition similar to that observed in the $S=\frac{1}{2}$ transverse Ising model

$$
H=\sum_{l=1}^{N}\left(S_{l}^{z} S_{l+1}^{z}-h S_{l}^{x}\right),
$$

at $T=0$ for $h \rightarrow h_{c}=\frac{1}{2}$. In this model, the GS changes from a singlet $\left(h>h_{c}\right)$ to a doublet $\left(h<h_{c}\right)$. It is important to note that at the critical field $h_{c}$, the excitation gap $\Delta$, which is nonzero for $h \neq h_{c}$, vanishes. The excitation gap must also disappear in the $S=1$ Heisenberg-Ising AFM at $\lambda=\lambda_{2}$ if the conclusions of $\mathrm{BJ}$ are correct, for it is easy to prove that a nonzero energy gap $\Delta$ between the GS and the excitation spectrum for the infinite chain is incompatible with the correlation function asymptotic behavior

$$
\left\langle S_{l}^{z} S_{l+R}^{z}\right\rangle \sim(-1)^{R} / R^{1 / 4},
$$

conjectured by BJ. Such a behavior implies that the wavenumber-dependent correlation function diverges at the Brillouin-zone boundary $\bar{q} \equiv q-\pi=0$ as

$$
\left\langle S_{q}^{z} S_{-q}^{z}\right\rangle \sim(\bar{q})^{-3 / 4} \text {. }
$$


Here, $\left\langle S_{q}^{z} S_{-q}^{z}\right\rangle$ is the integrated intensity

$$
\left\langle S_{q}^{z} S_{-q}^{z}\right\rangle \equiv \int_{0}^{\infty} \frac{d \omega}{2 \pi} S_{z z}(q, \omega)
$$

of the dynamic structure factor $S_{z z}(q, \omega)$ at $T=0$, from which we know that the first frequency moment $K_{z z}(q)$ is a linear function of $\cos q .^{29,30}$

$$
K_{z z}(q)=\int_{0}^{\infty} \frac{d \omega}{2 \pi} \omega S_{z z}(q, \omega)=A+B \cos q,
$$

with $|A|,|B| \leqslant \frac{1}{4}$. If we assume the presence of an energy gap $\Delta \geqslant 0$, Eqs. (9) and (10) imply for $\bar{q} \neq 0$

$$
\begin{aligned}
& \int_{0}^{\infty} \frac{d \omega}{2 \pi} \omega S_{z z}(q, \omega) \geqslant \Delta \int_{0}^{\infty} \frac{d \omega}{2 \pi} S_{z z}(q, \omega), \\
& \text { i.e., } \\
& A+B \cos q \geqslant\left\langle S_{q}^{z} S_{-q}^{z}\right\rangle .
\end{aligned}
$$

Since the left-hand side of (12) is bounded for all $q$ and the right-hand side diverges for $\bar{q} \rightarrow 0$ according to (8), the rigorous inequality (11) holds only if $\Delta=0$, i.e., the excitation gap is zero.

Finite-chain studies on the transverse Ising model were performed some time ago. ${ }^{31}$ It was found that the energies of a number of low-lying excited states measured from the GS energy have minima at $h \simeq h_{c}$ which become increasingly sharp as $N$ increases, reflecting the closure of the excitation gap at $h=h_{c}$ in the infinite system. No such effect has been observed for the $S=\frac{1}{2}$ Heisenberg-Ising AFM (1) as a function of $\lambda$ at $\lambda=1$, where a phase transition of completely different nature takes place (the excitation gap stays zero on one side of the transition). Hence it appears to be important to study the $\lambda$ dependence of the energies of low-lying excited states of the $S=1$ Heisenberg-Ising AFM (1) by finite-chain calculations in order to gain further insight into the exact location and into the nature of the $T=0$ phase transition expected at $\lambda \simeq 1$.

\section{SUMMARY AND DISCUSSION}

Botet and Jullien have recently performed a finite-size scaling analysis of spin-1 finite-chain calculations of $N=2$ through 12 spins. Their conclusions support a conjecture by Haldane that integral-spin Heisenberg-Ising AFM chains show different phase behavior from half-integral-spin Heisenberg AFM chains. Specifically, BJ conclude from their analysis that a new "intermediate" phase with a nonordered singlet GS and an excitation gap occurs for an extended range of anisotropy $\lambda$, including the Heisenberg point $\lambda=1$ [see Fig. 1(b)]. Since such a result is in apparent conflict with continuity and symmetry considerations, we have attempted to test the reliability of this numerical study by performing an analogous finite-size scaling analysis on $S=\frac{1}{2}$ finite chains for $N=2$ through 12 spins. The spin- $\frac{1}{2}$ Heisenberg-Ising AFM chain is known rigorously not to display such an "intermediate" phase [see Fig. 1(a)]. For easy-plane anisotropy $(0 \leqslant \lambda<1)$ the system is gapless with a singlet GS, and for easy-axis anisotropy $(\lambda>1)$, the GS is a doublet with an excitation-energy gap to a continuum of excited states. At the Heisenberg point $\lambda=1$, there is an essential singularity representing the termination of the gapless phase. However, our numerical studies on spin- $\frac{1}{2}$ finite chains reveal a picture strikingly similar to that found by BJ for spin 1. The arguments of BJ could easily lead to the spurious conclusion that the spin- $\frac{1}{2}$ Heisenberg AFM also shows an additional intermediate phase. We interpret the misleading results obtained by finite-size scaling techniques as being attributable to an essential singularity at the Heisenberg point for spin $\frac{1}{2}$. A singularity at the Heisenberg point for spin 1 could therefore also result in misleading results.

The analysis of BJ implies that the spin-1 excitation spectrum for $N \rightarrow \infty$ has the form sketched in Fig. 1(b). In particular, a continuum of excited states should "touch down" and close up with the GS at the point $\lambda_{2}$. A more detailed spin-1 analysis taking more of the excited states into account should provide further insight. Botet and Jullien remark that they hope to extend their analysis to spin$\frac{3}{2}$ and spin-2 chains. A finite-size analysis of spin- $\frac{3}{2}$ chains should be particularly revealing in the context of the above discussion.

In summary, the fact that the finite-size scaling behavior of spin- $\frac{1}{2}$ Heisenberg-Ising AFM chains so closely resembles the corresponding behavior of spin-1 chains, must cast doubt on the Botet-Jullien analysis. By implication, doubt is also cast on Haldane's contention that integer-spin Heisenberg-Ising AFM chains have very different phase behavior in the GS from half-integer-spin chains. Nevertheless, the existing numerical analyses on spin- $\frac{1}{2}$ and spin-1 systems are very intriguing. Further comparative studies on both spin- $\frac{1}{2}$ and spin-1 linear systems are very desirable, since it appears that the establishment (or otherwise) of the Haldane conjecture by numerical techniques requires great care and a more extensive numerical analysis. Finally, our preliminary study of the spin- $\frac{1}{2}$ AFM $X X Z$ model has revealed a number of interesting and unexpected results in the context of finite-size scaling analysis.

\section{Notes added in proof}

1. After this Comment was submitted for publication, we were informed by J. Sólyom that he had independently observed the fact that a finite-size scaling plot of spin- $\frac{1}{2}$ chains was qualitatively similar to the Botet-Jullien spin-1 plot, and had inferred that quite long chains were required to see the true asymptotic behavior. We are indebted to J. Sólyom for kindly sending some of his unpublished material.

2. We are pleased to see that R. Botet and R. Jullien and their associate E. W. Kolb have discussed the points made in this Comment in a paper submitted after our Comment was accepted, and published in J. Phys. A $\underline{16}$, L673 (1983). This paper is essentially an expanded version of the rebuttal which accompanies our Comment.

\section{ACKNOWLEDGMENTS}

We are indebted to Barry McCoy, Katarina Uzelac, and Vic Emery for stimulating discussions. This work was supported in part by National Science Foundation (NSF) Grant No. DMR-80-10819, in part by NSF Grant No. PHY-8109110 , and in part by the U.S. Department of Energy under Contract No. DE-AC02-76CH00016. One of us (J.C.B) acknowledges the hospitality offered during visits to the Institute for Theoretical Physics at The State University of New York, Stony Brook, and to Brookhaven National Laboratory. 
${ }^{1}$ F. D. M. Haldane, Institut Lau-Langevin Report No. SP81/95 (unpublished).

2F. D. M. Haldane, Bull. Am. Phys. Soc. 27, 181 (1982).

${ }^{3}$ F. D. M. Haldane, Phys. Lett. 93A, 464 (1983); Phys. Rev. Lett. 50, 1153 (1983).

${ }^{4}$ R. Botet and R. Jullien, Phys. Rev. B 27, 613 (1983).

${ }^{5}$ C. N. Yang and C. P. Yang, Phys. Rev. 151, 258 (1966).

${ }^{6}$ J. D. Johnson, S. Krinsky, and B. M. McCoy, Phys. Rev. A $\underline{8}, 2526$ (1973).

${ }^{7}$ J. des Cloizeaux and M. Gaudin, J. Math Phys. 7, 1384 (1966).

${ }^{8}$ A. Luther and I. Peschel, Phys. Rev. B 12, 3908 (1975); F. D. M. Haldane, Phys. Rev. Lett. 45, 1358 (1980).

${ }^{9}$ R. J. Baxter, J. Phys. C $\underline{6}$, L94 (1973); J. Stat. Phys. 9, 145 (1973).

${ }^{10}$ This result is trivial to prove for all $S$, since the Néel states are direct products of single-site states $\left|S, S_{l}^{z}\right\rangle$, with $S_{l}^{z}=(-1)^{l} S$ and $S_{l}^{z}=(-1)^{l+1} S$, respectively.

${ }^{11}$ R. B. Griffiths, Phys. Rev. Lett. 24, 1479 (1970). See also Critical Phenomena in Alloys, Magnets and Superconductors, edited by R. E. Mills, E. Ascher, and R. I. Jaffee (McGraw-Hill, New York, 1971), p. 377.

${ }^{12}$ L. P. Kadanoff, in Critical Phenomena, Proceedings of the International School of Physics "Enrico Fermi," Course LI, edited by M. S. Green (Academic, New York 1971), p. 100.

${ }^{13}$ P. W. Kasteleijn, Physica 18,104 (1952); T. W. Ruijgrok and S. Rodriguez, Phys. Rev. $\underline{119}, 596$ (1960); T. Oguchi, Phys. Rev. Lett. 11, 266 (1963); H. L. Davis, Phys. Rev. 120, 789 (1960).

${ }^{14}$ J. C. Bonner and M. E. Fisher, Phys. Rev. 135, A640 (1964).

${ }^{15}$ R. Orbach, Phys. Rev. $\underline{112}$, 309 (1958); L. R. Walker, ibid. $\underline{116}$, 1089 (1959); J. D. Johnson and B. M. McCoy, Phys. Rev. A $\underline{6}$, 1613 (1972).

${ }^{16}$ This should not be confused with the fact that in the context of the more general $X Y Z$ model

$$
H=J \sum_{l}\left[(1+\gamma) S_{l}^{x} S_{l+1}^{x}+(1-\gamma) S_{l}^{y} S_{l+1}^{y}+\lambda S F S I_{+1}^{z}\right],
$$

the whole line $|\lambda| \leqslant 1, \gamma=0$ is expected to be a line of singularities for $T=0$ properties. This has, however, only been verified for $S=\frac{1}{2}$. See Ref. 6 and R. J. Baxter, Ann. Phys. (N.Y.) $\underline{70}$, 323 (1972).

${ }^{17}$ C.-Y. Weng, thesis, Carnegie-Mellon University, 1968 (unpublished); T. de Neef, Phys. Rev. B $\underline{13}, 4141$ (1976).
${ }^{18}$ H. W. J. Blöte, Physica B $\underline{79}, 427$ (1975). Blöte analyzed his extrapolated results on the basis of spin-wave theory, but emphasized that this was an assumption.

${ }^{19}$ R. Kubo, Phys. Rev. 87, 568 (1952).

${ }^{20}$ It is important to distinguish between the gap $\Delta$ between the GS and the lowest excited states of the infinite system (sketched in Fig. 1) and the gap $G_{N}$ between the energies of the two lowest eigenstates of the finite system. In situations where the GS becomes degenerate in the thermodynamic limit, the $G_{N}$ arise from splitting of components of the GS by finite-size effects, and are consequently unrelated to the excitation gap $\Delta . \Delta$ is then the limit of a different sequence of finite-system gaps $\Delta_{N}$. The analysis of Ref. 4 does not reflect this important point.

${ }^{21}$ In fact, the "scaled gaps" must eventually diverge as $N G_{N} \sim N$.

${ }^{22}$ The value $\lambda=1.18$ is an extrapolation to the limit $N \rightarrow \infty$ of the successive crossing of the $N G_{N}$ curves for $N=4,6,8,10,12$. See Fig. 1 of Ref 4.

${ }^{23}$ H. W. J. Blöte (unpublished work). See also Ref. 18.

${ }^{24}$ T. Schneider, U. Glaus, and E. P. Stoll (unpublished); C. J. Hamer and M. N. Barber, J. Phys. A 14,241 (1981).

${ }^{25}$ Note that these gaps are related to the energy gap $\Delta$ of the infinite system for $\lambda>1$, where they are properly denoted $N \Delta_{N}$.

${ }^{26}$ H. H. Roomany and H. W. Wyld, Phys. Rev. D 21, 3341 (1980); Phys. Rev. B 23, 1357 (1981); see also C. H. Hamer and M. N. Barber, J. Phys. A 14, 259 (1981); M. P. Nightingale, J. Appl. Phys. 53, 7927 (1982).

${ }^{27}$ It should be pointed out that all the conclusions drawn in Ref. 14 in connection with Fig. 24 turned out to be correct, although a naive interpretation would have led to false predictions.

${ }^{28} \mathrm{An}$ apparent difference between $S=\frac{1}{2}$ and $S=1$ may be related to a difference in behavior of the excitation gap $\Delta$. For $S=\frac{1}{2}, \Delta$ increases exponentially slowly for $\lambda>1$ [see Eq. (4)]. For $S>\frac{1}{2}$ there is reason to suspect that $\Delta$ increases much more rapidly for $\lambda>1$ and this is likely to manifest itself as an apparent divergence of $N G_{N}$ at $\lambda=1$ for small $N$. J. C. Bonner, thesis, University of London, 1968.

${ }^{29}$ P. C. Hohenberg and W. F. Brinkman, Phys. Rev. B $\underline{10}, 128$ (1976).

${ }^{30}$ G. Müller, Phys. Rev. B 26, 1311 (1982).

${ }^{31} \mathrm{~J}$. Kurmann (unpublished work). 